\title{
Tactile Roughness: Neural Codes That Account for Psychophysical Magnitude Estimates
}

\author{
C. E. Connor, S. S. Hsiao, J. R. Phillips, ${ }^{a}$ and K. O. Johnson \\ Department of Neuroscience, The Johns Hopkins University School of Medicine, Baltimore, Maryland 21205
}

Hypothetical neural codes underlying the sensation of tactile roughness were investigated in a combined psychophysical and neurophysiological study. The stimulus set consisted of plastic surfaces embossed with dot arrays of varying dot diameter and center-to-center spacing. Human subjects explored each surface with the pad of the index finger and reported their subjective sense of roughness magnitude. The same surfaces were scanned across the receptive fields of cutaneous mechanoreceptive afferents in monkeys while recording the evoked action potentials. Hypothetical neural codes for roughness magnitude were computed from the neural response patterns and tested for their ability to account for the psychophysical data. The psychophysical results showed that subjective roughness magnitude is an inverted $\mathrm{U}$-shaped function of dot spacing that peaks near $3.0 \mathrm{~mm}$ spacing, and that increased dot diameter produces decreased roughness sensations at all dot spacings. Hypothetical neural codes that do not bear a consistent relationship to roughness magnitude across all of these stimulus conditions can be rejected as the code for roughness. Four types of neural codes were considered. They were based on (1) mean firing rate, (2) general variation in firing rate, (3) short-term temporal variation in firing rate, and (4) local spatial variation in firing rate. Mean firing rate failed to explain the psychophysical results: surfaces that evoked the same firing rate often evoked very different roughness judgments. In contrast, neural codes based on firing-rate variation, especially in slowly adapting afferents, account for the psychophysical results.

Roughness is one component of a larger perceptual category, texture, which plays an important role in vision, audition, and touch. Tactile texture perception is poorly understood; with few cxccptions, little is known about its dimensionality, its physical determinants, or its neural mechanisms. Roughness, one of the components of tactile texture that has been studied in some detail (Meenes and Zigler, 1923; Stevens and Harris, 1962; Culbert and Stellwagen, 1963; Ekman et al., 1965; Stone, 1967; Krueger, 1970; Cussler et al., 1977; Inukai et al., 1980; Green, 1981; Lederman, 1982; Sathian et al., 1989), is apparently unidi-

\footnotetext{
Received Apr. 5, 1990; revised Aug. 2, 1990; accepted Aug. 9, 1990.

This work was supported by NIH Grant R01 NS18787. We wish to thank James Vaccaro, who produced the stimulus surfaces used in this study.

Correspondence should be addressed to Kenneth O. Johnson, Department of Neuroscience, The Johns Hopkins University School of Medicine, 725 North Wolfe Street, Baltimore, MD 21205.

a Present address: University Laboratory of Physiology, University of Oxford, Parksroad, Oxford, UK OX1 3PT.
}

Copyright (C) 1990 Society for Neuroscience $0270-6474 / 90 / 123823-14 \$ 03.00 / 0$ mensional, because comparative magnitude judgments are possible. Further, the subjective sense of roughness satisfies the requirements of a ratio scale because subjects give consistent responses when asked to rate the relative roughness of different surfaces (Stevens and Harris, 1962).

Roughness has been defined qualitatively as the sensation that occurs when a nonuniform, 2-dimensional pattern is scanned across the skin surface (Meenes and Zigler, 1923). However, the exact physical determinants of roughness have remained elusive. Quantitative psychophysical studies of roughness using surfaces like sandpaper, metal gratings, and fabrics have related subjective magnitude estimates to physical parameters of the surfaces. The general result is that, within a range of spatial periods up to about $2.0 \mathrm{~mm}$, subjective roughness magnitude increases monotonically with increasing spatial period (Stevens and Harris, 1962; Lederman and Taylor, 1972; LaMotte, 1977; Green, 1981; Sathian et al., 1989). However, roughness is not just a simple function of spatial period. Experiments in which the spatial period of metal gratings was altered by independently varying groove width and bar width showed that groove width is the primary determinant of perceived roughness for such surfaces; increasing bar width actually decreases perceived roughness (Lederman and Taylor, 1972). Attempts to model the exact mathematical relationship between spatial form and roughness magnitude have met with only partial success (Taylor and Lederman, 1975). Some researchers have argued that roughness is not related to spatial form directly, but rather to the coefficient of friction between the skin and the stimulus surface (Ekman et al., 1965). However, changes in the coefficient of friction appear to have no effect as long as the spatial form of the stimulus surface remains constant (Taylor and Lederman, 1975). Thus, roughness magnitude appears to be a complex function of the structure of a textured surface, though the form of that relationship is unknown.

Regardless of what stimulus parameters determine roughness, the issue to be addressed here is the neural basis of roughness perception. This problem was first investigated by LaMotte, who used fabric stimuli in combined psychophysical and neurophysiological experiments and found a monotonic relationship between perceived roughness and discharge rates evoked in periphcral mcchanorcceptor afferents (LaMotte, 1977). This finding suggested that roughness magnitude might be encoded at the peripheral nerve level in terms of mean impulse rate. A more recent study by Sathian and colleagues involving grating stimuli has shown that mean rate in peripheral afferents can account for changes in perceived roughness due to altered groove width but cannot account for changes due to altered bar width (Sathian et al., 1989).

The studies reported here were motivated by an observation 


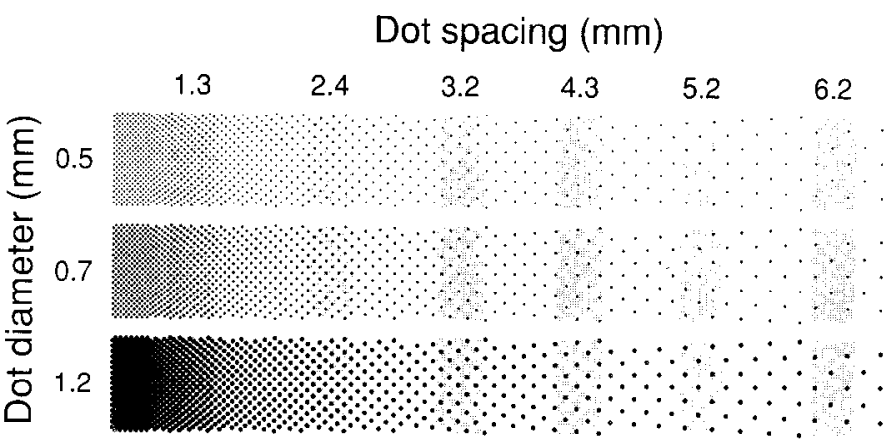

Figure 1. Stimulus surfaces used in these experiments. The surfaces consisted of dots (truncated cones) embossed upon the surface of a plastic sheet. Each solid circle in the figure represents the location of a single dot. Shaded areas represent regions that were used as test stimuli. The solid dark area represents a region in which the dot diameters cxceeded the distances between dot centers.

concerning the roughness sensations evoked by one set of surfaces that we routinely use in our neurophysiological experiments. These surfaces comprise embossed, tetragonal dot patterns, $212 \mathrm{~mm}$ long, in which the dot separation increases monotonically from $0.8 \mathrm{~mm}$ at one end to $6.5 \mathrm{~mm}$ at the other. The observation was that the surface felt quite smooth at the ends of the dot-spacing continuum but very rough in the middle, that is, that perceived roughness is an inverted $U$-shaped function of dot spacing. When we examined the mean impulse rates evoked by these surfaces, we found that mean rate also varied as an inverted U-shaped function of dot spacing. Under these conditions, a powerful form of logic can be applied. If a causal relationship is hypothesized between a particular neural code, for example, mean impulse rate, and the psychophysical response, then 2 stimuli that produce identical neural code values must also produce identical psychophysical values (allowing for differences due to random variation). This identity must exist in the original data; no subsequent transformation can produce it artificially.

The experiments described below were of 2 types. Psychophysical experiments were conducted with humans to obtain a quantitative characterization of the inverted U-shaped psychometric function relating perceived roughness magnitude to dot spacing. The diameter of the dots as well as their spacing was varied in order to increase the complexity of the psychometric data that would have to be explained by any candidate neural mechanism. Then, neurophysiological experiments were conducted in monkeys using the same dot patterns. Based on the assumption that the neurophysiological responses to dot patterns are similar in humans and monkeys (Johnson and Lamb, 1981; Phillips et al., 1990), a variety of neural coding mechanisms were tested for their ability to relate the psychophysical results to the neurophysiological responses.

Preliminary results were reported in a brief communication (Johnson et al., 1986).

\section{Materials and Methods}

Stimulus surfaces. The same embossed surface patterns were employed in the psychophysical and neurophysiological experiments. The method of surface production is described in Johnson and Phillips (1988). The patterns were $25 \mathrm{~mm}$ wide by $212 \mathrm{~mm}$ long and were composed of dots arranged in a square tetragonal pattern as illustrated in Figure 1. The center-to-center dot spacings, that is, the sides of the squares, were constant across the width of the pattern but increased linearly from 0.8 to $6.5 \mathrm{~mm}$ along its long $(212-\mathrm{mm})$ axis. Each dot had the form of a truncated cone with a flat top $350 \mu \mathrm{m}$ above the background and sides that sloped at an angle of $58-60^{\circ}$ with respect to the horizontal. Three patterns with dot diameters of $0.5,0.7$, and $1.2 \mathrm{~mm}$ (across the flat surface; base diameters were $0.4 \mathrm{~mm}$ larger) were produced and mounted side by side on a plastic drum so that dot spacing increased in the circumferential direction but remained constant in the axial direction, as illustrated in Figure 2 . In each of these 3 patterns, 6 segments were marked off for use as stimuli, as illustrated in Figure 1. Each segment spanned $11 \mathrm{~mm}$ along the circumferential dimension. The segments were positioned to produce mean center-to-center dot spacings of 1.3 , $2.4,3.2,4.3,5.2$, and $6.2 \mathrm{~mm}$. The resulting set of 18 stimulus patterns comprised 3 dot diameters at each of 6 center-to-center spacings.

Psychophysical methods. Subjects sat with the right arm resting on the surface of a table, the hand pronated and the index finger placed in a slot ( $35 \mathrm{~mm}$ long and $25 \mathrm{~mm}$ wide) that allowed lateral finger movements of 10-15 mm. On each trial, the subject lowered the finger to bring the finger pad in contact with one of the stimulus patterns and tactually explored the surface with whatever contact force and scanning velocity seemed most appropriate. The subject scanned each test surface along its short axis, that is, the axis with constant dot spacing. Between trials, the subject raised the finger to allow positioning of the next stimulus. Each of the 18 surfaces was presented 5 times to each subject in random order. In each trial, subjects reported their subjective estimate of the roughness of the surface by assigning a number to it. The surfaces were hidden from the subject's view at all times. The lack of any audible sound from the palpation, the interposition of a curtain between the subject and the surface, and relatively high ambient noise levels obviated the need for auditory masking. All of the plastic dot patterns had a smooth finish, and frictional sensations were minimized with talcum powder.

Roughness was not defined for the subjects. Instead, they were told to use their own concept of roughness from daily experience and report their sense of roughness magnitude as a number, using any numerical range that seemed appropriate. The only stipulation was that the number should be proportional to the sensation of roughness evoked by the stimulus. At the outset of the experiment, 10 representative surfaces (requiring no response) were presented in random order to give a sense of the range and to allow the subject to select a numerical scale.

Neurophysiological methods. Anesthetized macaque monkeys ( $\mathrm{Ma}$ caca mulatta) weighing between 3.0 and $5.0 \mathrm{~kg}$ were used. Single mechanoreceptive fibers were dissected from the median or ulnar nerves using methods described previously (Phillips and Johnson, 1981). Primary afferent fibers were classified (Talbot et al., 1968) as being slowly adapting (SA), rapidly adapting (RA), or Pacinian (PC) according to their responses to a vibrating punctate probe. Only fibers with receptive fields on the distal pad of one of the digits (D2-D5) were used. When a suitable fiber had been isolated, its receptive field was delineated with a small manual probe, and the most sensitive spot was marked with an ink dot. The drum with the stimulus surfaces attached was mounted on the hub of a mechanical stimulator, which is illustrated in Figure 2 and described in detail in Johnson and Phillips (1988). After manual positioning of the drum over the receptive field, the drum was lowered onto the skin with a controlled force, $30 \mathrm{gm}$, and rotated at an angular velocity corresponding to a drum surface speed of 20 or $50 \mathrm{~mm} / \mathrm{sec}$. After each revolution, the drum was shifted axially (at right angles to the direction of rotation) by $200 \mu \mathrm{m}$, thereby effectively causing the receptive field to scan the entire pattern in parallel sweeps displaced by $200 \mu \mathrm{m}$. All neurons were studied with at least 75 sweeps, which corresponds to a cumulative shift of at least $15 \mathrm{~mm}$ across the stimulus surface. The occurrence times of the action potentials evoked by the moving stimulus and of position codes emitted by a shaft encoder were recorded by the computer for later analysis and display. These shaft encoder pulses allow localization of the drum surface with an accuracy of $\pm 5 \mu \mathrm{m}$ (Johnson and Phillips, 1988)

The contact force, $30 \mathrm{gm}$, and scanning velocities, 20 and $50 \mathrm{~mm} /$ sec, used in the neurophysiological studies were chosen to be similar to those used by humans. Lederman (1974) showed that the natural, unrestrained force used by a subject in a roughness judgment task is about $100 \mathrm{gm}$. Contact forces of $30 \mathrm{gm}$ in the monkey and $100 \mathrm{gm}$ in the human produce approximately equal contact pressures because of the relative finger sizes. Timing of subjects' finger movements within the $10-15-\mathrm{mm}$ space in which the finger was allowed to move suggested that most subjects used scanning velocities of $20 \mathrm{~mm} / \mathrm{sec}$. Changes in these force and velocity values have little or no effect on roughness 
judgments and neurophysiological responses. Psychophysical studies by Lederman (1974) have shown that variations in force between 30 and $100 \mathrm{gm}$ and velocity between 10 and $250 \mathrm{~mm} / \mathrm{sec}$ have virtually no effect on roughness judgments. (It must be noted, however, that Lederman's experiments did not cover the full range of spatial periods studied here.) In neurophysiological studies using dot patterns, Johnson and Lamb (1981) showed that 3-fold variations in contact force from 20 to $60 \mathrm{gm}$ and 4-fold changes in scanning velocity from 40 to $160 \mathrm{~mm} / \mathrm{sec}$ produced only very small changes $(0-15 \%)$ in the spatial structure and impulse rate of the responses of monkey primary afferent fibers.

The stimulation methods in the neurophysiological experiments differ from those in the psychophysical experiments, where subjects actively scan the test surfaces. However, Lederman (1981) has shown that there is no difference in roughness judgments between trials in which a subject actively scans the test surfaces and those in which the surfaces are applied to his passive, restrained finger. This observation and experiments of our own comparing active and passive touch (F. Vega-Bermudez, S. S. Hsiao, and K. O. Johnson, unpublished observations) serve as justifications for our use of active touch in the psychophysical experiments and passive touch in the neurophysiological experiments (see also Discussion).

Analysis. Because subjects were allowed to choose their own numerical ranges for reporting roughness magnitude, the responses had to be normalized before combining data across subjects. The normalization consisted of dividing subjects' responses by their own grand averages, which resulted in an overall mean of 1.0 for cach subject. The normalized response values for each stimulus surface were averaged across subjects to produce the final 18 values used for comparison with the neural data.

The neural coding mechanisms based on temporal mechanisms were derived from a peristimulus-time histogram of instantaneous rate values. The instantaneous rate values were derived from a fractional-interval binning method that involved dividing the time axis into bins of constant duration, counting the number of intervals (including fractional intervals) in each bin, then dividing by the bin duration. In the test of each hypothetical coding mechanism, the bin duration was meant to approximate a central integration time, that is, the time period used for calculation of instantaneous impulse rate. The bin duration was constant within each analysis but was varied between analyses to assess the effect of this variable.

The spatial coding mechanisms were based on 2-dimensional ratc histograms, $R(x, y)$, which were derived as follows. First, a spatial event plot (SEP) was constructed for each neuron for each stimulus surface as described in Johnson and Phillips (1988). SEPs are raster plots in which each action potential is assigned an $(x, y)$ location based on the coincident location of the receptive field relative to the stimulus surface, $x$ being its circumferential location, and $y$, its axial location relative to a reference point on the drum surface. Then, $R(x, y)$, the instantaneous rate in impulses per second (ips), was calculated by dividing the SEP into spatial bins, counting the number of whole and fractional intervals in each bin, then dividing by the total dwell time in each bin. The bin size in the $y$ direction was maintained constant at $200 \mu \mathrm{m}$, the axial drum shift increment between sweeps. The $x$ bin size was fixed in any one analysis but varied between analyses.

The sources of variation in each response parameter were assessed with a 2-way analysis of variance (ANOVA) based on the model

$$
r_{i j}=\mu+S_{j}+N_{i}+e_{i j}
$$

where $r_{i j}$ represents the response of neuron $i$ to stimulus $j, \mu$ represents the grand mean response over all neurons in a single class and all stimuli, $S_{j}$ represents the mean effect of stimulus $j, N_{i}$ represents the difference between the $i$ th neuron's mean response to all stimuli and the grand mean, and $e_{i j}$ represents the unexplained variation. The unexplained variation consists of differences in (1) stimulus effects between neurons and (2) short-term, stochastic fluctuations that are part of all neuronal discharge sequences. The magnitude of this stochastic component could have been assessed most simply by running all of the stimuli at least twice and comparing differences in codes between separate runs; however, that was impractical because of the time required for 1 prescntation of all of the surfaces at 2 velocities. Instead, the action potentials from alternate sweeps were assigned to 2 separate SEPs, and the coding computation was performed on each of the SEPs. The result of this procedure is 2 SEPs and 2 computed code values for each of the 18 combinations of dot size and spacing. The 2 SEPs for each stimulus have the same structure as the original, except that each has a scanning resolution of $400 \mu \mathrm{m}$ instead of $200 \mu \mathrm{m}$, and each contains roughly half of the action

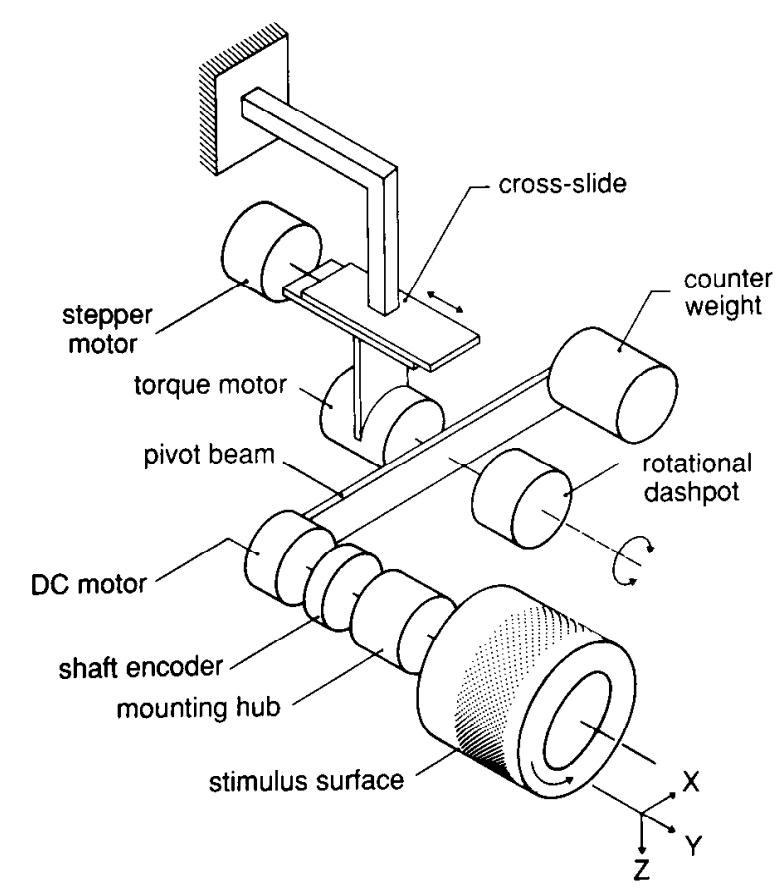

Figure 2. Rotating drum stimulator used to present stimuli in neurophysiological experiments. The stimulus surface is wrapped around a plastic drum and scanned across the skin by continuous rotation. On each revolution, the pattern is stepped $0.2 \mathrm{~mm}$ at right angles to the scanning direction, so that the skin-contact patch traces a series of parallel paths around the surface of the drum. The rotational and translational positions of the stimulus pattern are monitored by means of pulses emitted by a shaft encoder mounted on the drum shaft.

potentials. These SEPs differ from one another because of stochastic fluctuations and because the sweeps in one are shifted by $200 \mu \mathrm{m}$ relative to the other. The mean squared difference between the pairs of code values provides a worst-case estimate of the effect of discharge variability because it also includes differences that result from different scanning trajectories. The mean squared error calculated in this way is at least 4 times greater than the variance due to stochastic variation. $A$ factor of 2 derives from the fact that the variance of the difference of 2 independent measures is twice the variance of the individual measures. $\Lambda$ nother factor of 2 derives from the fact that all of the hypothetical response measures are means, and the variance of a mean based on half the sweeps through a test region is twice as great as a mean based on all of the sweeps (assuming statistical independence between sweeps). The effect of stochastic variability turned out to be miniscule for all hypothetical measures because of the large number of impulses factored into each computation. (A single $11 \times 15 \mathrm{~mm}$ patch of SEP contains approximately 2000 impulses at a mean rate of 50 impulses per sec.)

\section{Results}

\section{Psychophysical results}

The psychophysical results are summarized in Figure 3. The left panel shows curves for individual subjects at the $0.7-\mathrm{mm}$ dot diameter to illustrate the consistency between subjects. The standard deviation of responses to individual patterns ranged from 0.064 to 0.394 , though most were near 0.2 (root mean square deviation, 0.22 ). Cluster analysis showed that the subjects' responses formed a single homogeneous group. On this basis, the responses were averaged across subjects to give the results shown in the right panel ( 21 subjects, 14 male, 7 female). The normalized magnitude scale illustrated in Figure 3 spans a large sensory range. Subjectively, the roughest surfaces felt like 36-grit coarse sandpaper, while the smoothest surfaces felt like smooth plastic. Indeed, the largest dots $(1.2 \mathrm{~mm})$ at the smallest 
Figure 3. Psychophysical results. Normalized roughness magnitude is plotted against dot spacing in both panels. $A$, Curves for individual subjects (based on 5 presentations of each stimulus; dot diameter, $0.7 \mathrm{~mm}$ ). $B$, Average curves for the 3 dot diameters (based on 21 subjects).
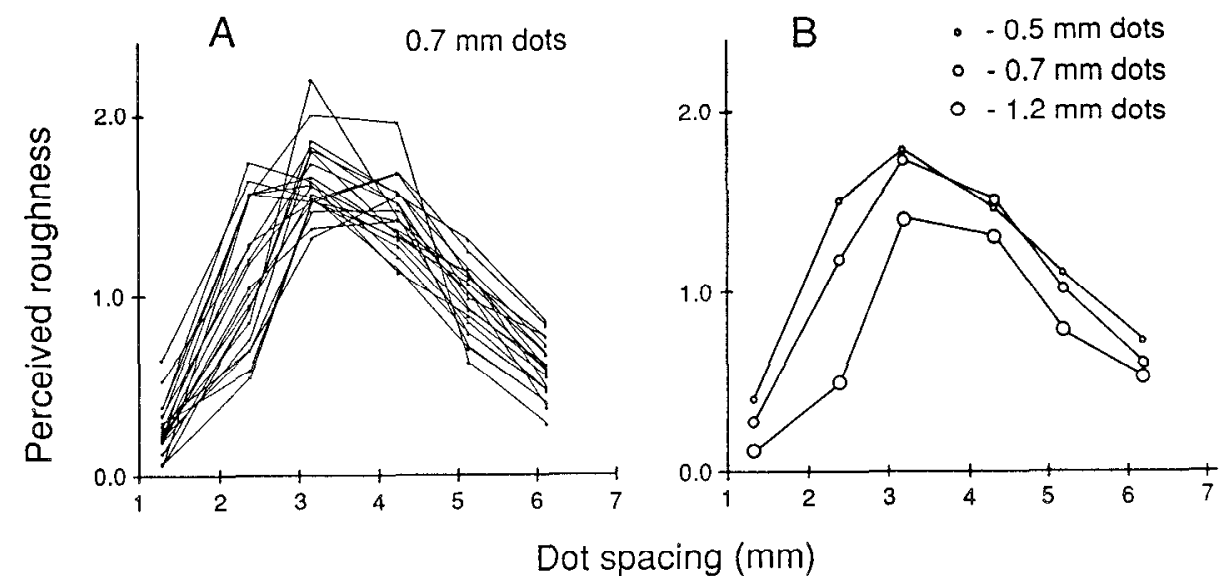

spacing $(1.3 \mathrm{~mm})$ come close to coalescing into a smooth, uninterrupted surface.

Both dot diameter and dot spacing had statistically significant effects on roughness estimates (ANOVA $p<0.0001$ ). Perceived roughness decreased somewhat with increasing dot size, especially at the closer dot spacings. Dot spacing had a much larger effect. At each dot diameter, roughness increased with increasing dot spacing up to $3.2 \mathrm{~mm}$, then declined. Thus, the roughness of these dot surfaces is a nonmonotonic function of dot spacing. Figure $3 B$ shows, for example, that dot spacings of approximately 2 and $5 \mathrm{~mm}$ produce the same roughness magnitude even though they evoke very different neural responses, as described in the next section. The remainder of the paper is concerned with discovering the coding mechanism that extracts a constant factor in different neural responses that evoke the same subjective sense of roughness.
Figure 4. SEP sections for typical SA, RA, and PC fibers. The corresponding sections of the stimulus pattern are shown in the top row (dot diameter, 0.5 $\mathrm{mm}$ ). Each SEP comprises a set of rasters resulting from repetitive parallel scans of the stimulus across the receptive field, with the stimulus shifted vertically by $0.2 \mathrm{~mm}$ between each scan.
SA

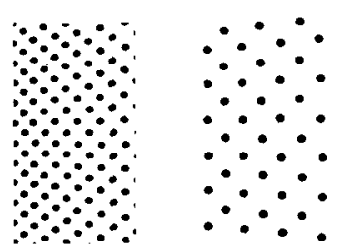

RA
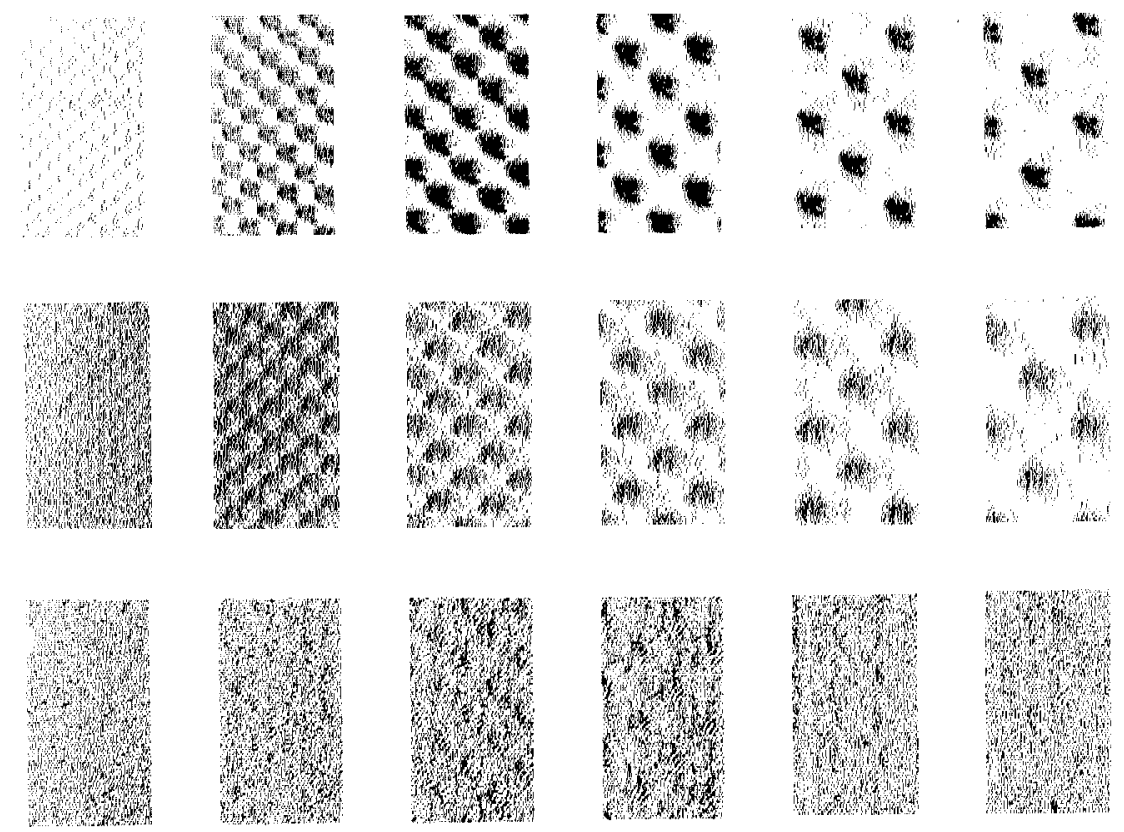

1.3
2.4

3.2
4.3

5.2

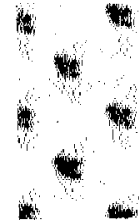

6.2

Dot spacing (mm) 
SA

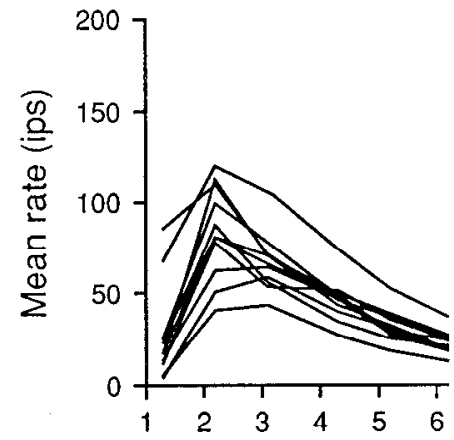

RA

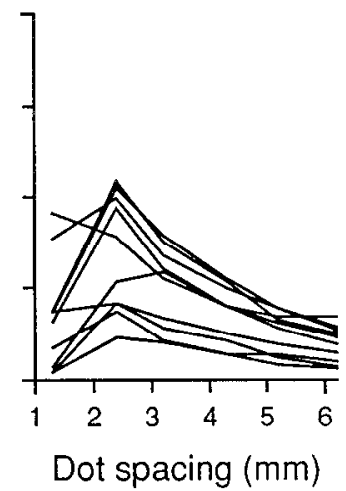

PC

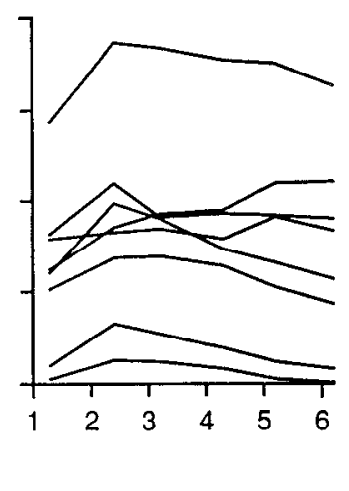

Figure 5. Mean firing rate as a function of dot spacing. Mean rate, averaged across rate histograms, is plotted in ips. Results for individual fibers are shown for each of the 3 afferent types. Dot diameter, $0.7 \mathrm{~mm}$.

\section{Neurophysiological results}

Neurophysiological data were collected from 28 SAs (cutaneous slowly adapting afferent fibers), 21 RAs (cutaneous rapidly adapting afferent fibers), and 11 PCs (deep rapidly adapting afferent fibers, i.c., Pacinian afferents) using tetragonal dot patterns like those used in the psychophysical studies. A subset of these neurons, 11 SAs, 10 RAs, and 8 PCs, was studied using the identical surfaces that had been presented to the human subjects in the psychophysical experiments, and all of the quantitative comparisons between neural and psychophysical results are based on these data. The larger set serves to verify that this subset provides a representative sample of each of the neuron types. The contact force and scanning velocity were $30 \mathrm{gm}$ and $20 \mathrm{~mm} / \mathrm{sec}$ (see Materials and Methods). Five SA neurons were also studied with a scanning velocity of $50 \mathrm{~mm} / \mathrm{sec}$. The analytical results for the 2 data sets were similar. Representative SEPs for each of the 3 fiber types at $20 \mathrm{~mm} / \mathrm{sec}$ are shown in Figure 4.

The effects of variability within and between neurons were analyzed for each of the hypothetical neural codes. The results for mean rate arc presented in somc detail because they are typical of the results for other codes. Figure 5 illustrates the relationship between mean rate and dot spacing for individual SA, RA, and PC afferents. SA responses showed the least variability between neurons; the RA responses showed somewhat greater variability. The $\mathrm{PC}$ responses were much more variable than either the SA or RA responses. The data were subjected to an ANOVA to ascertain the significance of the stimulus effects. The results of the ANOVA for mean rate are shown in Table 1. Systematic stimulus effects account for most of the SA response variance, less of the RA variance, and little of the PC variance; however, stimulus effects were statistically significant in each case. A separate analysis (see Materials and Methods) showed that stochastic variation has a minuscule effect relative to the other sources of variation. The standard error due to stochastic variation for single points illustrated in Figure 5 averages 0.22 ips for $\mathrm{S} \Lambda \mathrm{s}, 0.13$ ips for RAs, and 1.42 ips for PCs.

\section{Coding studies}

The neural codes that were tested yielded single values for each of the 18 stimulus surfaces. Each of these values is meant to approximate the output of a hypothetical central neural mechanism responsible for the subjective sense of roughness. The values generated by these codes were averaged across neurons using arithmetic and geometric means. Correlation coefficients between the neural and psychophysical data were generally higher for the arithmetic means, but the differences were small. The results presented below are based on arithmetic means.

Neural codes of 4 types were considered. The first hypothetical code to be tested was the mean impulse frequency evoked by each stimulus surfacc. This hypothcsis was tcstcd bccausc previous studies had suggested that roughness may be related to firing rate (e.g., LaMotte, 1977). Moreover, it was considered reasonable that an intensive sensation such as roughness might be encoded by the mean firing rate in 1 or more of the primary afferent fiber populations.

The second type of hypothetical code consisted of statistical measures of impulse frequency variation such as variance, standard deviation, and mean deviation. While it seems unlikely that mathematical algorithms of this type would be employed by the nervous system, they are useful for testing the general hypothesis that the nervous system extracts some related measure of impulse frequency variation. This hypothesis was tested on the assumption that roughness, which appears to depend on variation in the height profile of a palpated surface, might logically be encoded by variation in the impulse frequency of peripheral afferents.

\begin{tabular}{|c|c|c|c|c|c|c|}
\hline \multicolumn{2}{|c|}{$\begin{array}{l}\text { Source of } \\
\text { variation }\end{array}$} & \multirow{2}{*}{$\begin{array}{r}\begin{array}{c}\text { Sum of } \\
\text { squares }\end{array} \\
99,085\end{array}$} & \multirow{2}{*}{$\begin{array}{l}\text { Per- } \\
\text { cent } \\
\text { of } \\
\text { vari- } \\
\text { ation }\end{array}$} & \multirow{2}{*}{$\begin{array}{c}\begin{array}{c}\text { Degrees } \\
\text { freedom }\end{array} \\
17\end{array}$} & \multirow{2}{*}{$\begin{array}{r}\begin{array}{l}\text { Mean } \\
\text { square }\end{array} \\
5,828\end{array}$} & \multirow{2}{*}{$\begin{array}{l}F \text {-ratio } \\
\text { MS/MSE } \\
32.9^{* *}\end{array}$} \\
\hline SA & $\mathrm{S}$ & & & & & \\
\hline $\mathrm{SA}$ & $\mathrm{N}$ & 34,621 & 21 & 10 & 3,462 & $19.5^{* *}$ \\
\hline SA & $\mathrm{E}$ & 30,090 & 18 & 170 & 177 & \\
\hline $\mathrm{RA}$ & $S$ & 62,713 & 45 & 17 & 3,689 & $16.7^{* *}$ \\
\hline $\mathrm{RA}$ & $\mathrm{N}$ & 42,845 & 31 & 9 & 4,761 & $21.5^{* *}$ \\
\hline RA & $\mathrm{E}$ & 33,813 & 24 & 153 & 221 & \\
\hline $\mathrm{PC}$ & $\mathrm{S}$ & 14,977 & 5 & 17 & 881 & $3.4^{*}$ \\
\hline $\mathrm{PC}$ & $\mathrm{N}$ & 283,451 & 86 & 7 & 40,493 & $154.6^{* *}$ \\
\hline $\mathrm{PC}$ & $\mathrm{E}$ & 31,178 & 9 & 119 & 262 & \\
\hline
\end{tabular}

$S$ represents stimulus effects on mean rate, that is, changes in mean rate due to changes in dot spacing and dot diameter; $\mathrm{N}$ represents differences in mean rate between neurons; $E$ represents the residual variance, which is mainly due to differences in stimulus effects between neurons.

$* p<0.05$.

$* * p<0.01$. 

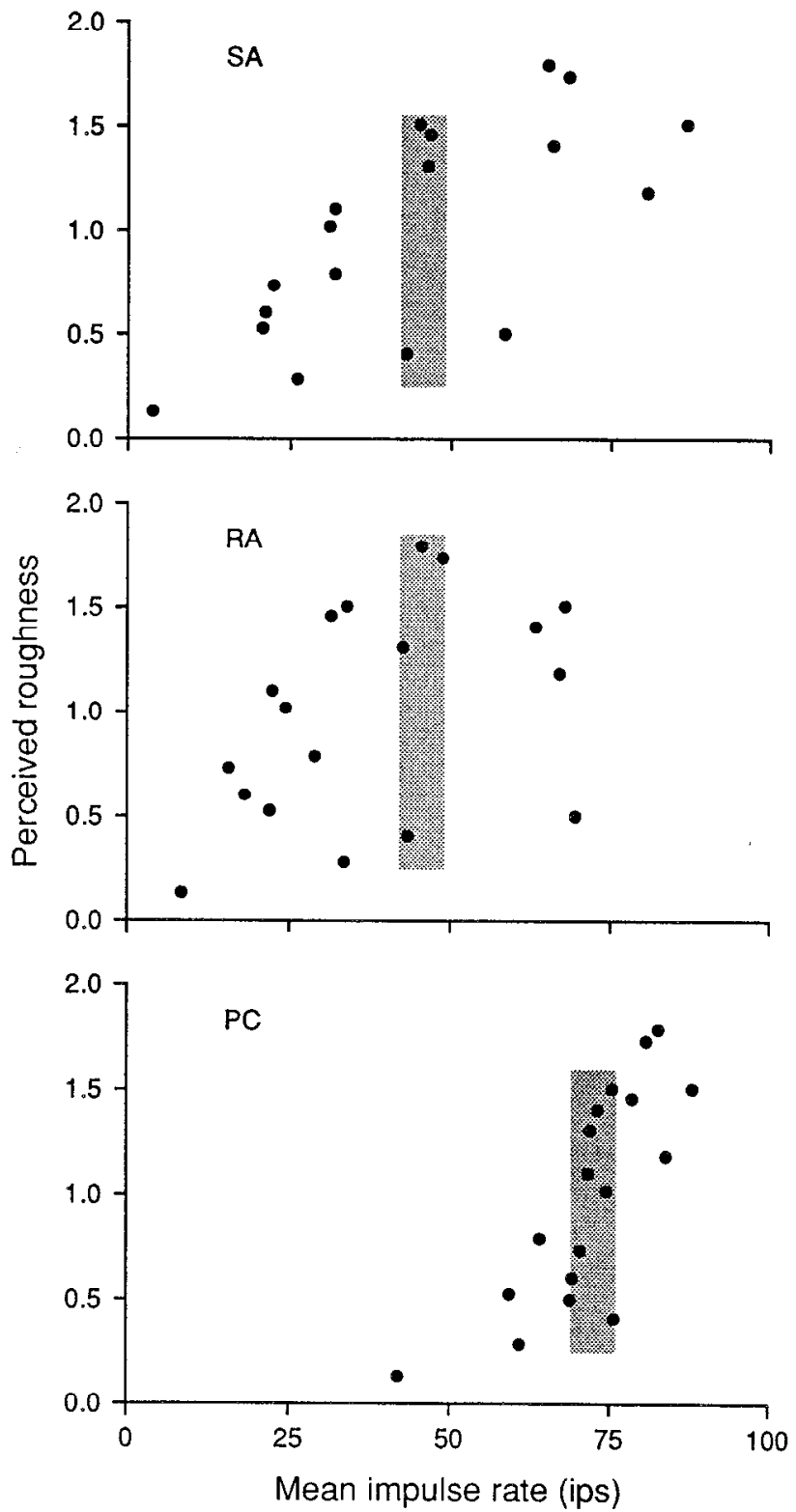

Figure 6. Subjective roughness versus mean firing rate. Normalized roughness magnitude, averaged across 21 subjects, is plotted against mean rate in spikes per second, averaged across 11 SA fibers, $10 \mathrm{RA}$ fibers, and $8 \mathrm{PC}$ fibers. Each point represents the mean impulse rate and roughness magnitude evoked by 1 of the 18 stimulus surfaces. The shaded areas represent points at $45 \pm 3$ ips for SA and RA afferents and $72 \pm 3$ ips for PC afferents.

Hypothetical codes of the third type were statistical measures of short-term temporal variation in firing rate. Temporal variation measures were based on changes in impulse frequency across specified time intervals. In comparison with the general firing-rate variation measures, temporal variation codes were used to test a similar but more specific hypothesis - that the sensation of roughness is based on short-term temporal fluctuations in the discharge rates of individual fibers summed across entire afferent populations. The rationale behind the temporal variation measures was that the nervous system might sense the roughness or unevenness of a surface through the intermittent stimulation of individual afferents as the surface moves across the skin. The greater the unevenness, the greater would be the temporal fluctuations in skin indentation and hence discharge rate.

The fourth type of hypothetical code consisted of 2-point measures of local spatial variation in firing rate. The hypothetical central mechanisms related to these measures would depend on firing rates in pairs of afferent fibers. Firing rates in pairs of afferents provide information about spatial gradients in firing rate across the skin surface at a scale determined by the distances between the afferents. Previous studies using scanned patterns have shown that the SA and RA populations transmit a moving image to the CNS that is closely approximated by the SEPs derived from single SA and RA afferent fibers (Phillips et al., 1988). The SAs and RAs innervate the skin densely and, within each class, respond in nearly identical ways to the same stimulus. The same discharge pattern simply passes from afferent to afferent as the stimulus moves over the skin, which creates a spatially consistent moving image such as the SEPs displayed in Figure 4. Thus, pairs of firing rates separated by a specified displacement in the SEP correspond to firing rates in pairs of afferents whose receptive fields are separated by the same displacement on the skin surface. Our 2-point spatial variation measures were based on pairwise differences in firing rate averaged over all positions and directions for a specific displacement. These measures were used to test the hypothesis that the nervous system perceives the roughness of a surface by means of the uneven pattern of stimulation delivered to the receptor sheet. The greater the roughness of the surface, the greater would be the local variation in skin indentation and hence the greater the variation in firing rate between nearby receptors.

\section{Mean firing rate}

Subjective roughness magnitude correlates poorly with mean firing rate. The roughness magnitude and mean impulse rate evoked by each surface are shown in Figure 6 for each of the 3 fiber types. Each point simultaneously represents the mean rate averaged across all fibers of 1 type for a single stimulus pattern and the corresponding average psychophysical magnitude. A causal relation between mean rate and roughness magnitude judgments would show up on this graph as some consistent (not necessarily linear) relationship between the 2 measures. No causal relationship is evident in Figure 6. On the contrary, nearly identical impulse rates are evoked by surfaces that produce some of the highest and some of the lowest roughness judgments. For example, SA rates near $45 \mathrm{ips,}$ which are enclosed in the shaded region, were associated with one of the smoothest surfaces (1.3 $\mathrm{mm}$ separation, $0.5 \mathrm{~mm}$ diameter) and one of the roughest (3.2 $\mathrm{mm}$ separation, $0.5 \mathrm{~mm}$ diameter). The shaded regions in the RA and PC graphs in Figure 6 illustrate similar inconsistencies. A neural code based on mean rate would produce identical roughness magnitudes for these stimuli rather than the very different magnitudes that were observed.

The lack of correlation cannot be attributed to variability within or between neurons or variability in the psychophysical magnitudes. Variations in the overall mean response rate between neurons cause the grand mean in Figure 6 to shift left or right but leave the relationships between points unaffected. Variations in the stimulus effects between neurons do cause scatter, but the rms standard errors due to this effect are only 4.0, 4.7, and 5.7 ips for the respective SA, RA, and PC means. The standard error of the psychophysical values averaged 0.049 . All of these sources of variation are small compared to the disparities illustrated in Figure 6. 


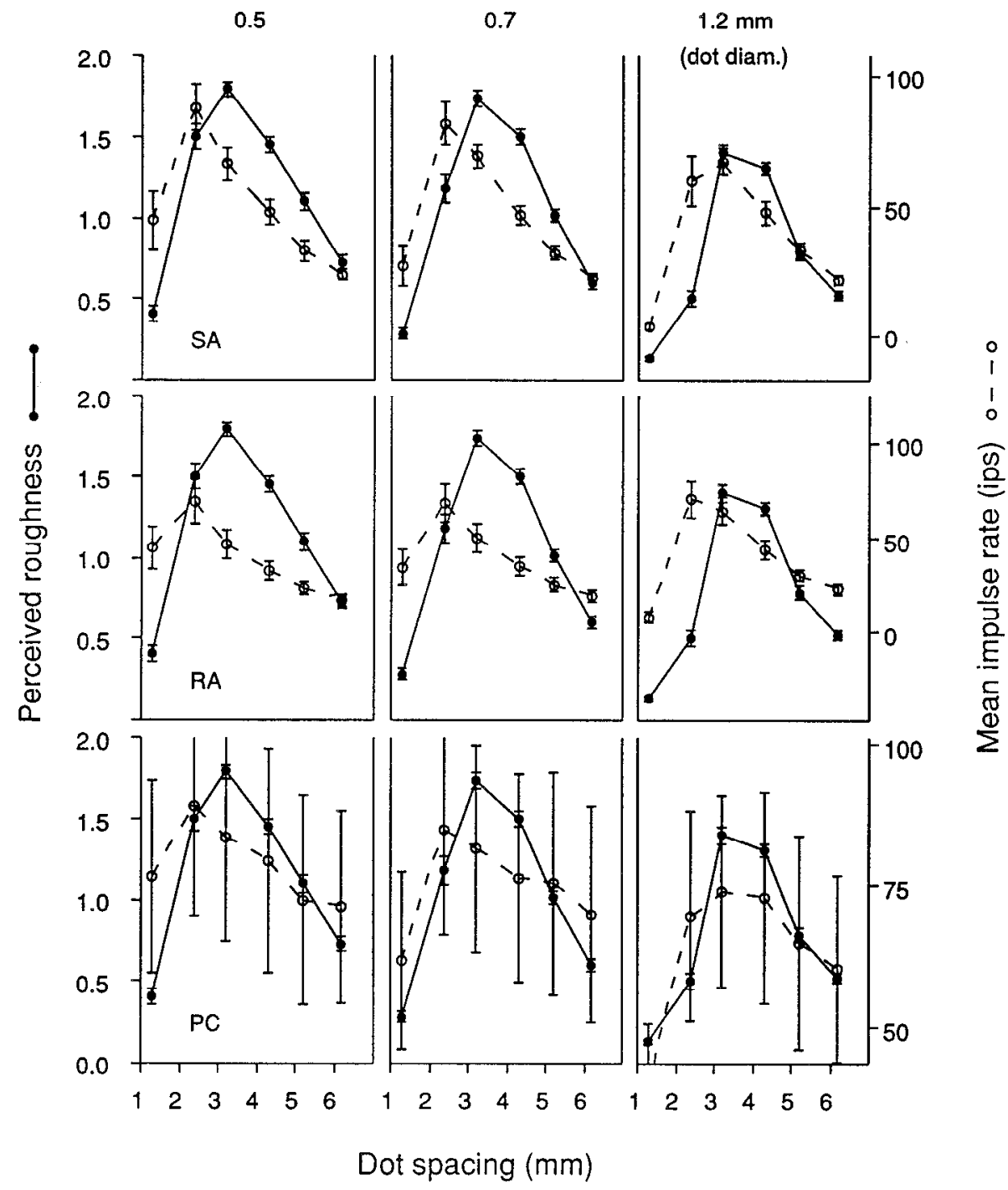

Figure 7. Mean impulse rate and roughness versus dot spacing. Mean roughness magnitude, which is represented on the left-hand ordinate, is symbolized by solid circles and continuous lines. Mean impulse rate, which is represented on the right-hand ordinate, is symbolized by open circles and dashed lines. Dot spacing is shown on the $a b$ scissa. The offset and scale of the righthand ordinate relative to the left-hand ordinate was obtained by a linear regression of roughness magnitude on mean impulse rate, which produces the mean squared best fit between the 2 sets of data. Results for SA, RA, and PC fibers are shown in the top, middle, and bottom rows, respectively. Results for the $0.5,0.7$, and $1.2 \mathrm{~mm}$ dots are shown in the left, middle, and right columns, respectively. Vertical bars indicate SEM for each point.
The same data are replotted in Figure 7 to show the systematic nature of the inconsistencies. Because there was no suggestion of a nonlinear relationship, a linear regression was used to compare the psychophysical and neural data. Figure 7 shows that mean firing rate peaks at a smaller dot spacing than does roughness magnitude: scaled mean fring rate is consistently higher than roughness magnitude at fine dot spacings and lower than roughness at middle and higher dot spacings. No simple linear combination of rates across afferent classes would improve the correlation because the mean rates for SAs, RAs, and PCs all err in the same manner. For these reasons, no further tests of coding mechanisms based on mean firing rate were undertaken.

\section{Firing rate variation}

As a group, measures of firing rate variation were better predictors of roughness magnitude than were mean rate measures. Three measures of variation were calculated: mean absolute deviation from the mean impulse rate (the first absolute central moment), variance, and standard deviation. These measures were computed for individual fibers and then averaged, as described previously. The final standard deviation values were the means of the square roots of the variances for individual fibers.
Subjects' roughness judgments are closely correlated with both SA mean deviation and SA variance, as can be seen from the correlation coefficients in Table 2. Roughness judgments are less correlated with the RA and PC data. The product moment correlations listed in Table 2 are good indices of covariation for mean deviation and variance, as there were no evident nonlinear trends between these measures and the psychophysical judgments. However, because the relationship between variance and roughness is linear, a nonlinear relation between standard deviation and roughness is expected. Positive curvature was in

Table 2. Correlation coefficients of roughness judgments versus mean rate and rate variation measures

\begin{tabular}{llll} 
& SA & RA & PC \\
\hline Mean rate & 0.710 & 0.429 & 0.801 \\
Variance & 0.970 & 0.922 & 0.554 \\
Mean deviation & 0.974 & 0.865 & 0.848 \\
Standard deviation $^{a}$ & 0.933 & 0.914 & 0.856
\end{tabular}

a Based on $\log$-log transformation of roughness judgments and the standard deviation of rate. 

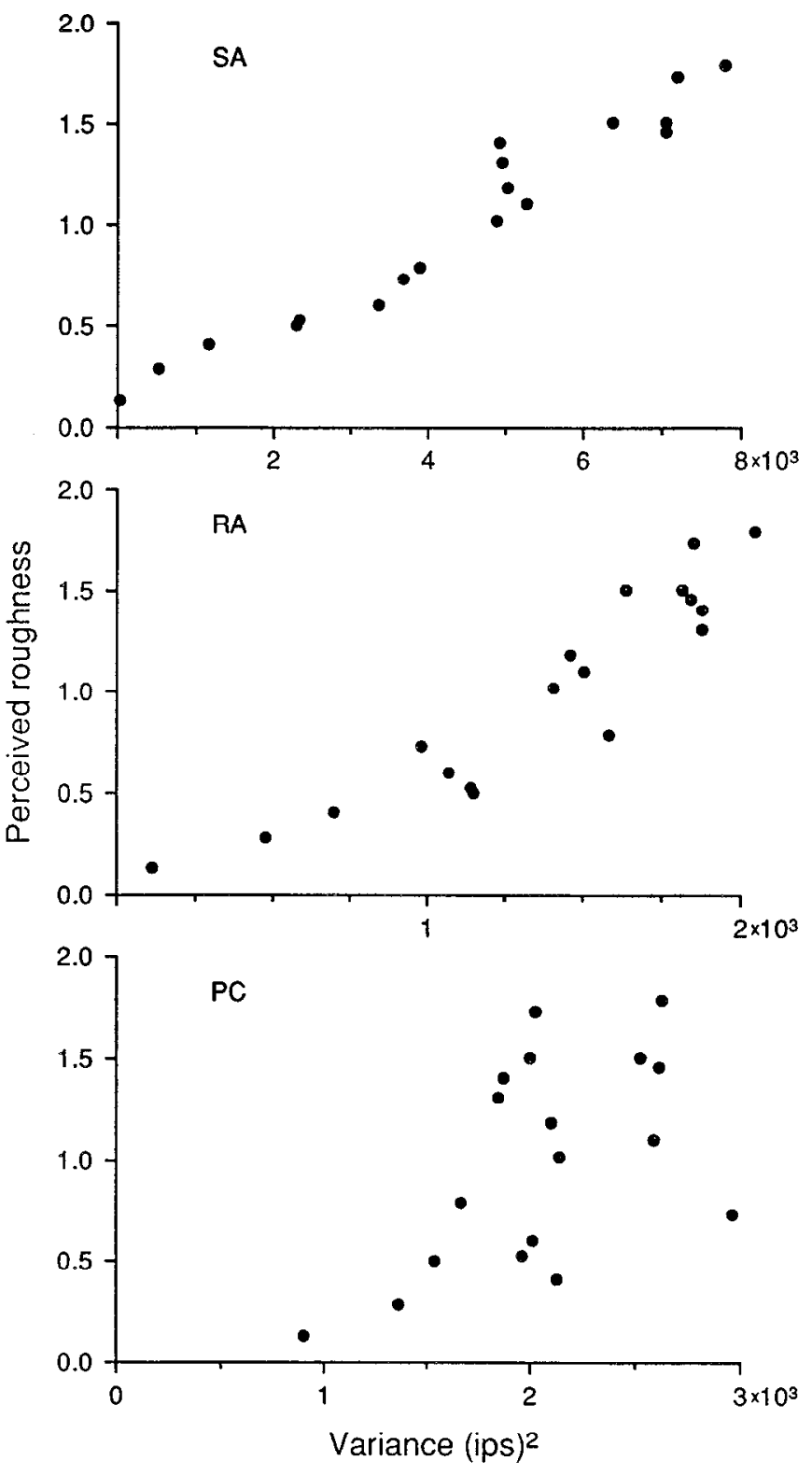

Figure 8. Subjective roughness versus firing rate variance. Details are as in Figure 6.

fact observed, and the data were transformed to $\log -\log$ coordinates before calculating the correlation coefficient shown in Table 2 . The effect of bin size was tested by varying the bin size used for calculating the individual impulse rates. The correlations between these measures and subjective roughness are relatively constant across a range of bin sizes from 0.5 to $30 \mathrm{msec}$, then drop at longer integration times.

Impulse rate variance, shown in Figure 8 for each of the fiber classes, was chosen for illustration. The remarkable improvement in consistency (relative to a mean rate code) for the SA and RA afferents can be seen by comparing Figures 6 and 8 . Because, as in the rate data, there was no suggestion of nonlinearity in the covariation plots, linear regressions were used to scale the neurophysiological data illustrated in Figure 9. Both the SA and $R A$ variance values, but particularly the $S A$ variance values, match the psychophysical roughness judgments well. There are no systematic differences between the $\mathrm{SA}$ variance values and the psychophysical judgments, except for a small underestimate of the roughness judgments produced by patterns with dot spacings near $3.0 \mathrm{~mm}$. In contrast, the RA variance values systematically exaggerate the roughness of the $1.2-\mathrm{mm}$-diameter dots, yielding higher predicted roughness values than for the 0.5 - and $0.7-\mathrm{mm}$ dots. This effect is the reverse of that observed in the psychophysical experiments. The lack of consistency between $\mathrm{PC}$ variance and the psychophysical values is evident in both Figures 8 and 9.

$\mathrm{SA}$ and, to a lesser extent, RA rate-variation measures apparently capture some aspect of the neural discharge that is closely related to roughness judgments. However, it should be emphasized that these measures are not proposed as specific neural mechanisms. Variation measures of this kind are in fact unsuitable as neural codes because they take no account of the spatial or temporal scale of firing rate variation. Codes based on these measures can lead to anomalous results when firing rates vary on a large spatial or temporal scale (cf. Rosenfeld and Kak, 1982, p 295). The remainder of the paper concerns hypothetical codes that depend upon local temporal or spatial variation.

\section{Temporal variation}

Local temporal variation was measured by calculating firing rate differences for all pairs of bins separated by a time delay, $d t$. These firing rate differences were used to calculate statistics such as the mean absolute difference and the mean squared difference in firing rate (cf. Haralick et al., 1973). These statistics yielded results that were similar to those reported for the general measures of variation. Mean absolute difference was chosen to illustrate temporal variation because its correlation values were slightly higher than for the other measures.

Subjective roughness magnitude is highly correlated with SA temporal variation, as can be seen in Figure 10. The SA correlation is 0.973 at the optimum delay value of $130 \mathrm{msec}$. The RA and PC correlations are lower $(0.922$ and 0.865 , respectively). As before, linear regressions were used to scale the 2 sets of values and produce the graphs shown in Figure 11 . These graphs show that there is no systematic difference between SA temporal variation and subjective roughness. $\mathrm{RA}$ temporal variation, like RA impulse rate variance, also predicts the dotspacing effects well but fails to predict the effects of dot diameter. At each dot spacing of $3.0 \mathrm{~mm}$ and higher, the effects of changes in dot diameter on RA temporal variation are inconsistent with their effects on subjects' roughness judgments: increased dot diameter lowers the subjective magnitude but raises the RA temporal variation. The $\mathrm{PC}$ values are least closely related to subjective roughness.

The results in Figures 10 and 11 are based on rate histograms with a $10-\mathrm{msec}$ bin width. As with the general variation measures, correlation coefficients were relatively constant across a range of bin widths up to about $30 \mathrm{msec}$, then fell gradually with increasing bin widths. A more critical parameter was the temporal delay value, that is, the time interval across which rate differences were being measured. Correlation for SA temporal variation at $20 \mathrm{~mm} / \mathrm{sec}$ was highest at $130 \mathrm{msec}$. Figure 12 illustrates the way in which correlation declined at lower and higher delay values. These results suggest that subjective roughness is most closely related to fluctuations in SA firing rate on a scale of about 100-150 msec. At a scanning velocity of 20 $\mathrm{mm} / \mathrm{sec}$, this range corresponds to a distance of travel of 2-3 $\mathrm{mm}$. (The optimum delay of $130 \mathrm{msec}$ corresponds to $2.6 \mathrm{~mm}$.) 


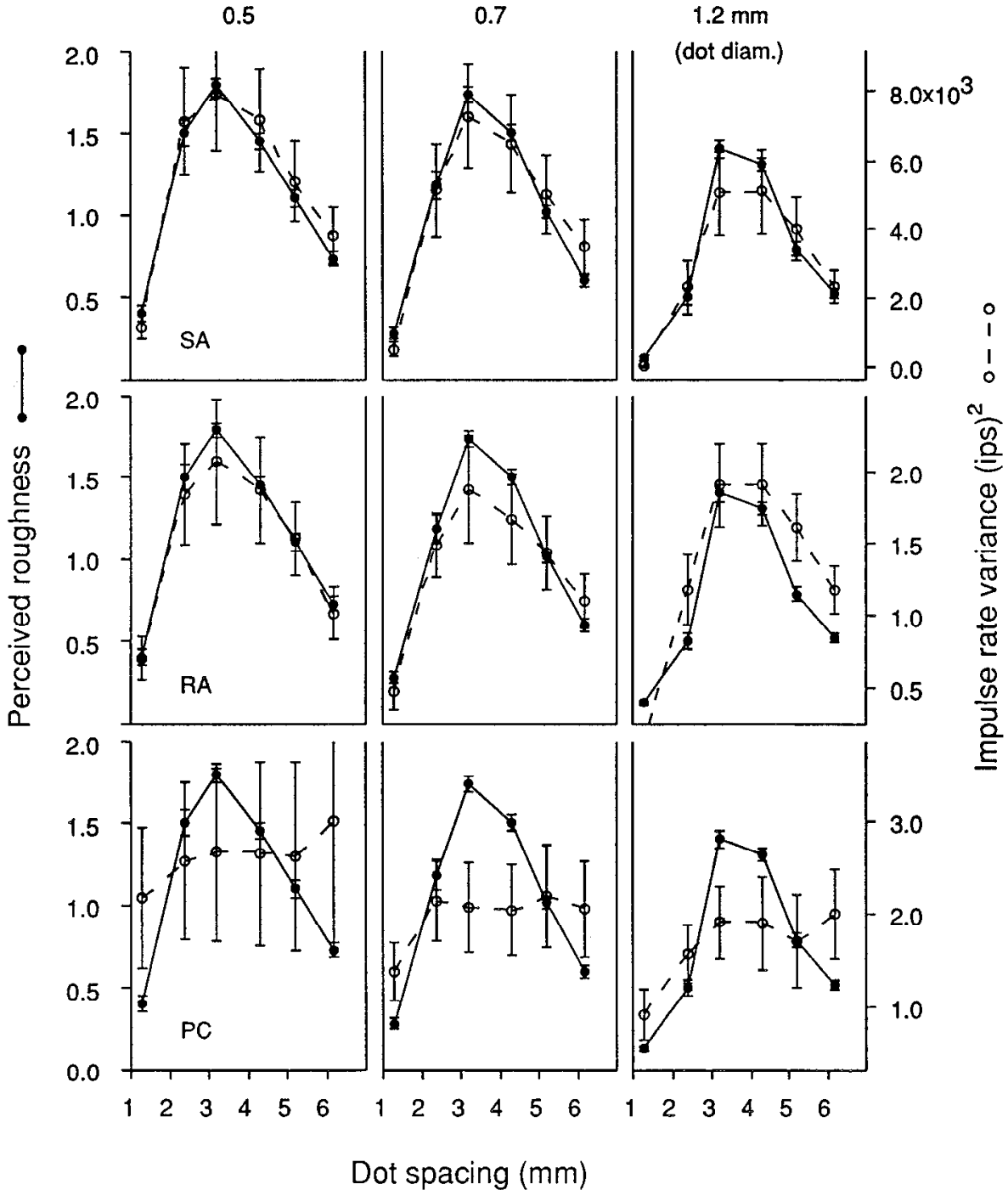

Figure 9. Firing rate variance and roughness versus dot spacing. Details are as in Figure 7.
The question arises as to which is actually the critical value, temporal delay or spatial distance. The simplest neural mechanism for measuring temporal variation would depend on a fixed $d t$. On the other hand, the finding that roughness estimates are constant across a range of scanning speeds (Lederman, 1974) suggests that any temporal mechanism would need to be adjusted to maintain some kind of spatial constancy. This issue was addressed by analyzing the responses of a subset of the SA fibers studied at a scanning velocity of $50 \mathrm{~mm} / \mathrm{sec}$. The results, which are displayed in Figure 12, suggest that the optimum delay period shows constancy for space rather than time: at $50 \mathrm{~mm} /$ $\mathrm{sec}$, the corrclation was highest for a delay of $44 \mathrm{msec}$, which corresponds to $2.2 \mathrm{~mm}$ of travel, close to the $2.6 \mathrm{~mm}$ optimum at $20 \mathrm{~mm} / \mathrm{sec}$.

\section{Spatial variation}

Measures of local spatial variation were based on firing-rate gradients in SEPs. These measures reflect firing-rate variations across the afferent populations innervating the 2-dimensional skin surface, as described above. Local spatial variation was measured by calculating firing rate differences for pairs of bins separated by a distance, $d$. As with temporal variation, these firing-rate differences were used to calculate a number of statistics. Mean absolute difference is chosen for presentation because it gave the highest correlation values. The formula used in calculating this statistic is

spatial variation

$=\frac{1}{12} \sum_{a=15}^{180} \frac{1}{n x \cdot n y} \sum_{x=1}^{n x} \sum_{y=1}^{n y}|[R(x+d x, y+d y)-R(x, y)]|$,

where $a$ is the angle between bin pairs, which varies between 15 and $180^{\circ}$ in $15^{\circ}$ increments, $n x$ and $n y$ represent the number of pairs within the analysis interval, and $d x$ and $d y$ represent displacements in the $x$ and $y$ directions:

$$
\begin{aligned}
& d x=d \cdot \cos (a) \\
& d y=d \cdot \sin (a) .
\end{aligned}
$$

This formula is based on Haralick et al. (1973).

The results for spatial variation are similar to those for temporal variation and are shown in Figure 13. The correspondence for the SA afferents is improved marginally relative to SA temporal variation; the $\mathrm{SA}$ correlation is 0.983 at an optimum 

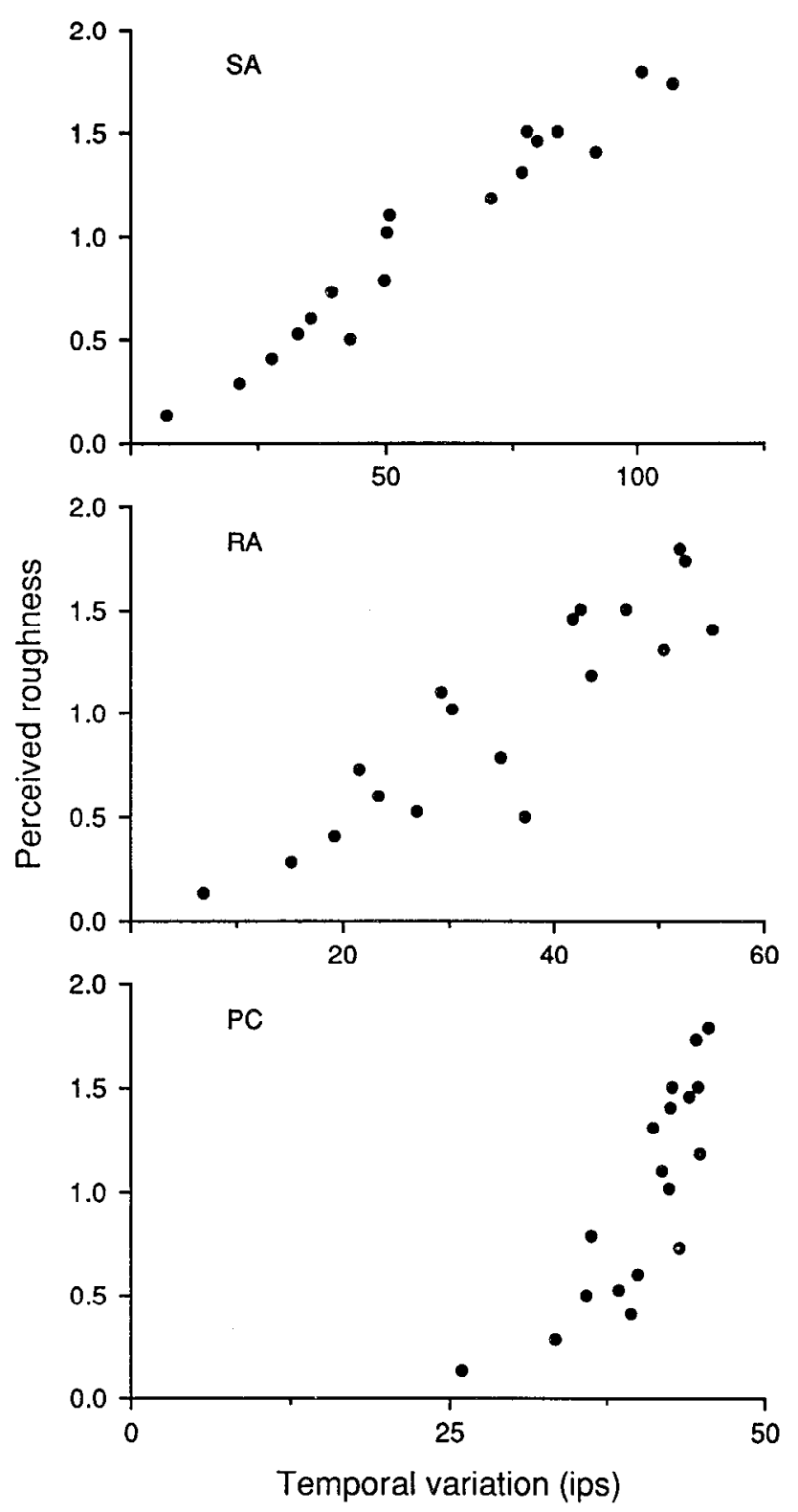

Figure 10. Subjective roughness versus temporal variation. Details are as in Figure 6.

displacement, $d$, of $2.2 \mathrm{~mm}$. The RA and PC correlation coefficients are 0.901 and 0.793 with optimum displacements of 2.0 and $1.6 \mathrm{~mm}$, respectively. The data are replotted in Figure 14, using linear regressions to scale the ordinates. As before, SA spatial variation matches the psychophysical data without any systematic differences. The RAs respond to increasing dot size with increasing spatial variation, which is the reverse of the psychophysical effect.

The rate values were calculated from 10 -msec bins, but, as before, this parameter had little effect when varied from 0.5 to 30 msec. Correlation coefficients for SA afferents peaked at a spatial displacement of $2.2 \mathrm{~mm}$ and declined at lower and higher values (see Fig. 15). This suggests that, if roughness is based on spatial variations in firing rate, variations on a scale of about 2 $\mathrm{mm}$ are critical. This distance corresponds to approximately 2 receptor spacings in the finger pad of humans and monkeys (Johansson and Vallbo, 1979; Darian-Smith and Kenins, 1980).

\section{Discussion}

\section{Psychophysical results}

The novel psychophysical result reported in this paper is the decline of perceived roughness for spatial periods greater than $3.2 \mathrm{~mm}$. No previous study (of which we are aware) has reported this decline, presumably because previous studies have been confined to stimuli with spatial periods less than $3.0 \mathrm{~mm}$. Most of what is known about roughness for surfaces with shorter spatial periods comes from the studies of Lederman and her colleagues (Lederman and Taylor, 1972; Lederman, 1974, 1981; Taylor and Lederman, 1975), who have studied roughness sensations evoked by metal gratings with gaps and ridges $1.0 \mathrm{~mm}$ or less in width. Roughness magnitude for these surfaces was shown to increase as a near linear function of gap width over the range from 0.125 to $1.0 \mathrm{~mm}$ and to decline with increasing ridge width over the same range. Sathian et al. (1989) extended the range of gap widths $(0.2-1.7 \mathrm{~mm})$ and got the same result, that is, a near linear rise in subjective magnitude with increasing gap width and a drop with increasing ridge width. The 6 surfaces in our study with center-to-center spacings of 1.3 and $2.4 \mathrm{~mm}$ have gaps between dots of $0.1,0.6,0.8,1.2,1.7$, and $1.9 \mathrm{~mm}$. Our results over this range also show a linear relationship between roughness and gap size and a decline with increased element size. However, at larger spacings, we found a decline in perceived roughness.

Besides gratings, the stimulus most commonly used for studying perceived roughness is sandpaper. Experiments with sandpaper are more difficult to interpret because of the random nature of the stimulus structure. The general result is that subjects' roughness judgments rise with increasing coarseness over a wide range of grit numbers (24-320; grit $=$ number of meshes per linear inch in the grading sieve). The relationship can generally be expressed as a power law in terms of inverse grit number with an exponent near 1.0 (Stone, 1967; Green, 1981) or slightly greater than 1.0 (Stevens and Harris, 1962). Examination of these sandpapers under a microscope suggests that the mean spacing between particles is approximately 3 times the grit size, for example, $3 \mathrm{~mm}$ for the coarsest sandpaper used in these studies. Thus, the results of studies using sandpaper appear to be consistent with our results and those of Lederman and Taylor (1972) and Sathian et al. (1989).

\section{Neural mechanisms of roughness perception}

Hypothesis testing based on the comparison of human psychophysical judgments with monkey neural-response measures is valid only if the neural responses are similar in the 2 species. There is extensive literature on the psychophysics and neurophysiology of tactual sensation in humans and monkeys, which has tended to show the similarity of the 2 species. The only quantitative psychophysical study using exactly the same stimuli in humans and monkeys (Mountcastle et al., 1972) showed that vibratory detection and discrimination thresholds are similar in the 2 species. The only published study using scanned dot patterns in humans (Phillips et al., 1990) has shown that SEPs of the responses of SA (human SAI), RA, and PC afferents to Braille patterns are similar in the 2 species (cf. Johnson and Lamb, 1981). Thus, we assume that the SA, RA, and PC responses obtained in the current study are similar to those that would have been obtained if the neurophysiological experiments had been done in humans. In contrast, human SAII afferents yield SEPs unlike any that have been observed in the monkey. 


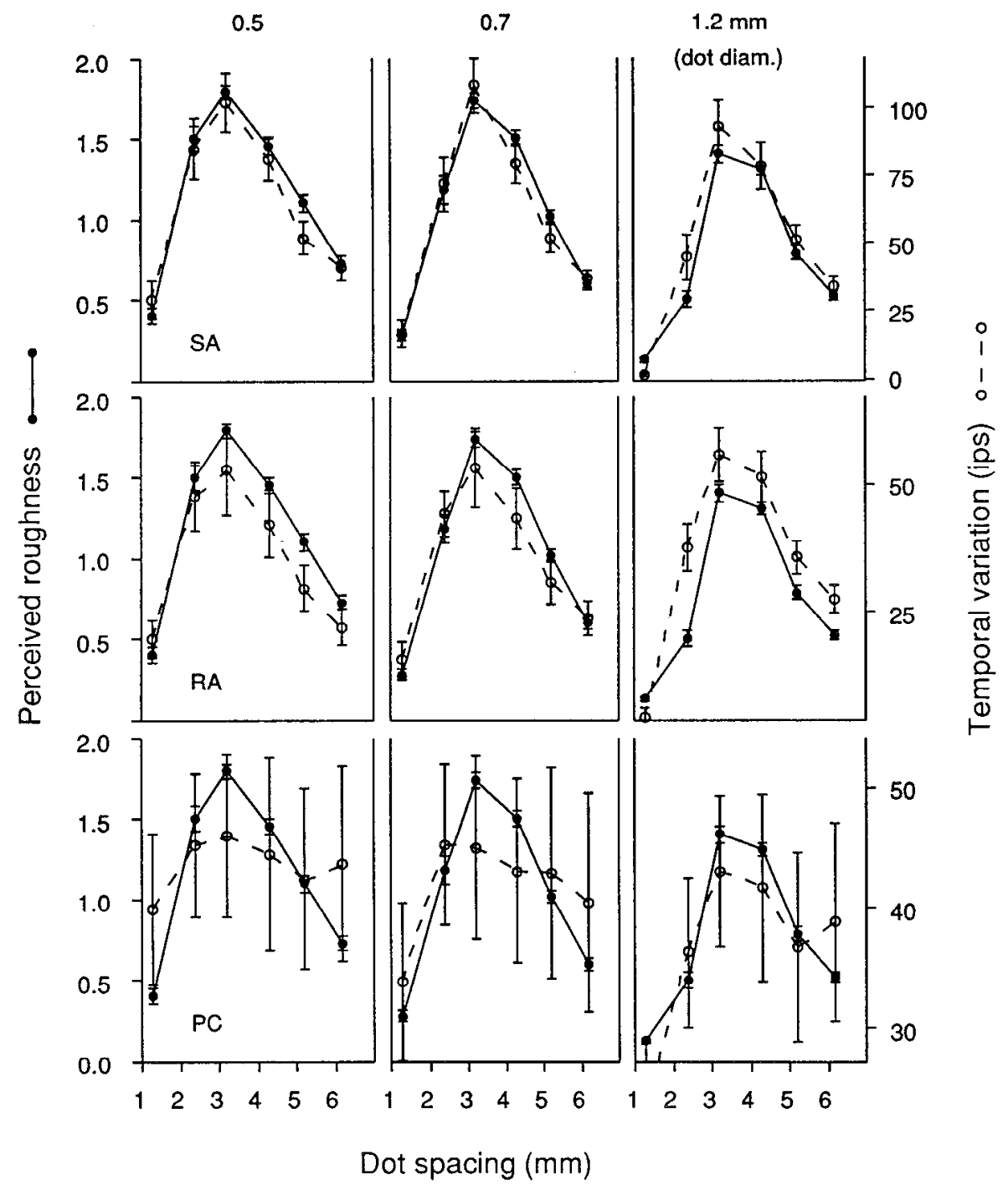

Figure 11. Temporal variation and roughness versus dot spacing. Details are as in Figure 7.
However, there are 2 arguments against the possibility that SAII afferents are responsible for roughness magnitude judgements. First, the discharge rate of SAII afferents is primarily sensitive to tangential skin stretch. Thus, the demonstration by Taylor and Lederman (1975) that tangential skin force has no effect on roughness magnitude would seem to rule out SAII mean ratc as a code for roughness. Second, SAII afferents exhibit poor spatiotemporal resolution (Phillips et al., 1990), and the evidence suggests strongly that roughness magnitude depends on the spatial structure of the stimulus as it is manifested in the spatiotemporal structure of the afferent discharge (as discussed below).

The first hypothesis that we tested was that roughness is based on mean impulse rate in 1 or more of the afferent fiber populations. Our results suggest that mean rate cannot account for the roughness of the patterns used in this experiment. Mean rate has been evaluated as a hypothetical code for roughness in 2 previous studies (LaMotte, 1977; Sathian et al., 1989). The surfaces used in those studies were not like the ones used here, but we would judge them to be no coarser than our dot pattern with 2.4-mm dot separations. LaMotte (1977) reported a mono-

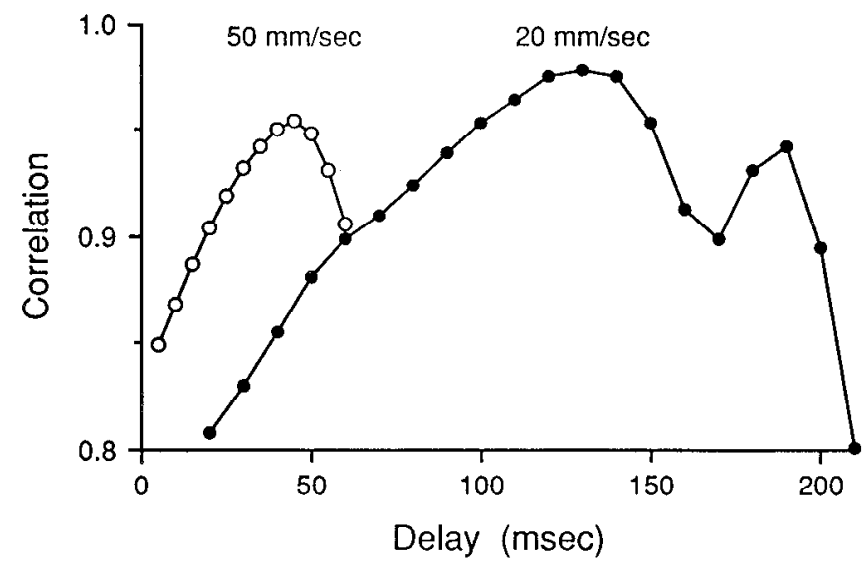

Figure 12. Correlation between roughness and SA temporal variation versus delay interval. Each point represents the correlation coefficient between subjective roughness and SA temporal variation based on a specific delay interval, which is specified on the abscissa. 

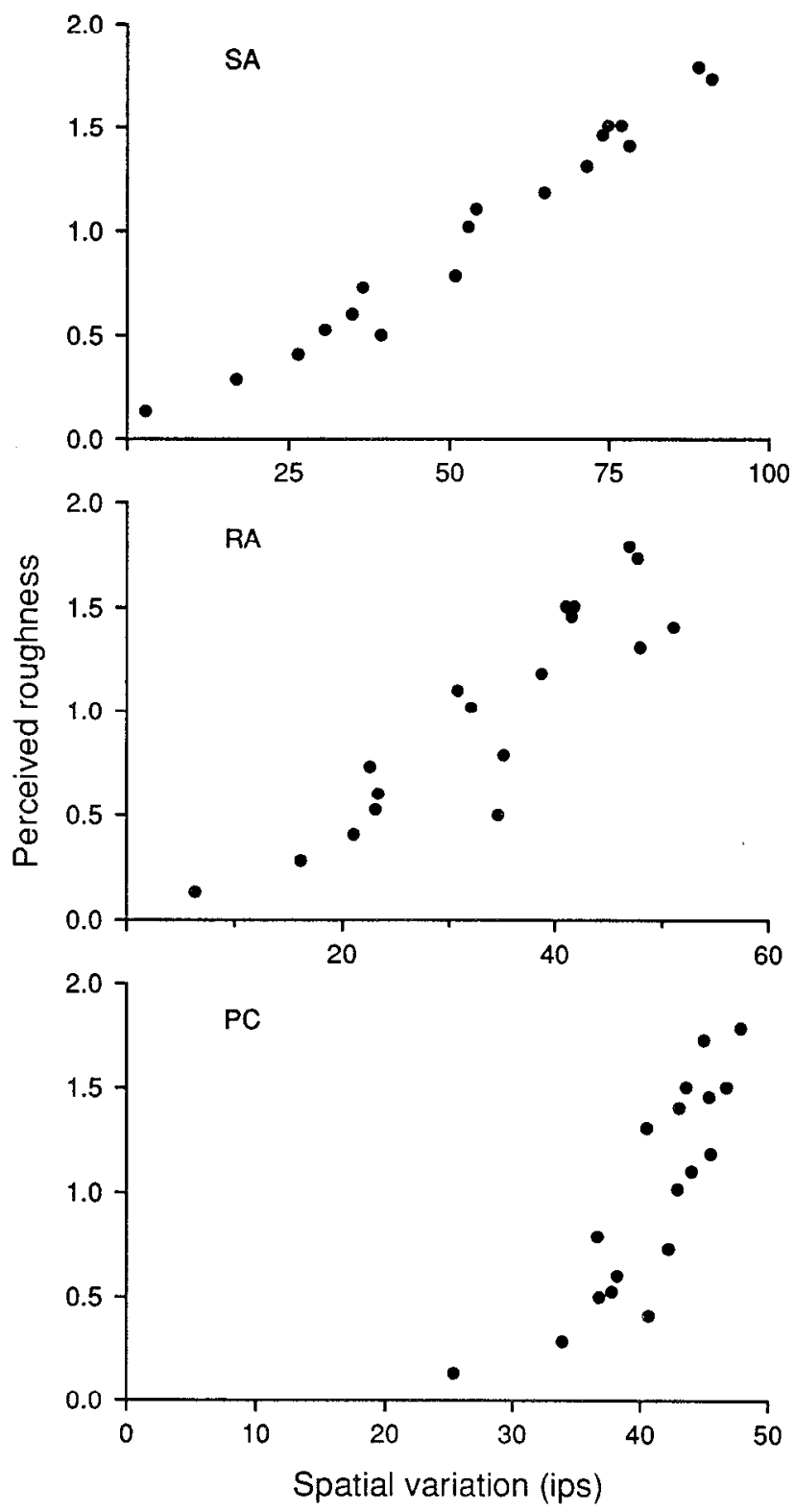

Figure 13. Subjective roughness versus 2-point spatial variation. Details are as in Figure 6.

tonic covariation between roughness judgments and RA mean rate. Sathian et al. (1989) reported a monotonic covariation only for the PC afferents. If we were to confine our analysis to the 1.3- and 2.4-mm dot separations, we would report a monotonic covariation only for the SA afferents. Taken together, these studies cover a wide range of stimulus conditions, and no simple hypothesis based on mean rate accounts for the results. Moreover, when our analysis is extended to larger spatial periods, stimuli that evoke identical mean firing rates are judged to have very different roughness magnitudes.

The remaining hypotheses were based on the subjective observation that roughness seems to be related to stimulus-intensity variation. Meenes and Zigler (1923) said, "Roughness is a tactual perception ... characterized by a lack of uniformity of stimulation [and] by several levels of intensity [that] are constantly undergoing intensive change." Therefore, our second hypothesis was that roughness is based on some measure of variation in firing rate. The statistics examined at this stage were mean deviation, variance, and standard deviation of impulse rate. Each of these measures, when applied to SA responses, produced a close correlation with the psychophysical results.

The high correlations produced by the general statistical variation measures led us to test more specific hypotheses having to do with firing-rate variation. In particular, we postulated that roughness might be based cither on temporal variation or on spatial variation in firing rate. Both of these hypotheses are based on mean differences in firing rate between pairs of points separated in space or time. The temporal variation measures model neural mechanisms that compare firing rates at pairs of time points separated by a fixed delay. These measures produced close correlations with subjective roughness, especially in the case of SA afferents. The match was quite sensitive to the delay magnitude, and the optimal delay value varied with scanning velocity. However, the optimal delay did correspond to a constant 2-mm distance of travel at both $20-$ and $50-\mathrm{mm} / \mathrm{sec}$ scanning velocity. If roughness perception depends on temporal variation, then the neural mechanism must compensate for changes in scanning velocity.

The spatial variation measures model neural mechanisms that compare firing rates between afferent fibers whose receptive field centers are separated by a fixed distance. Subjective roughncss was most highly correlated with firing-rate differences between SA afferents. Separation between fibers was critical: maximum correlation was obtained by assuming a distance of $2.2 \mathrm{~mm}$ between afferent fibers. This distance is close to 2 primary afferent spacings in the skin of humans and monkeys (Johansson and Vallbo, 1979; Darian-Smith and Kenins, 1980).

The spatial variation results suggest a fairly simple type of physiological mechanism. The mechanism would require higher-level neurons with receptive fields that contain excitatory and inhibitory subregions separated by about 2 afferent spacings. Such neurons would respond in proportion to the difference in input level between the antagonistic subregions. An entire population of such neurons would effectively measure the same thing as the spatial variation algorithm, that is, the average difference in firing rate between neighboring points in the receptor sheet. Thus, the mean firing rate in such a population would be proportional to roughness magnitude and could serve as the neural basis for the sensation of roughness.

The coding studies discussed above were limited to surfaces with dot spacings of $1.3 \mathrm{~mm}$ and greater. The finest sandpapers used in roughness studies ( 320 grit) have grit spacings on the order of $200 \mu \mathrm{m}$, which is much finer than the smallest dot spacing used in this study. It is questionable whether a spatial variation code could account for the perceived roughness of such surfaces. Johnson (1983) noted this coding problem and suggested that the relative impulse rates in SA, RA, and PC afferents might provide the code for the roughness of finer surfaces. A systematic study of roughness coding for surfaces with spatial periods less than $1.0 \mathrm{~mm}$ is required.

\section{Visual and auditory roughness}

Texture is a dimension not only of tactile sensation but of visual and auditory sensation as well. In vision research, variability measures of the sort used in the present study have been successfully applied in digital image processing for the purpose of automatic discrimination between textures in photographs (Haralick et al., 1973). However, an attempt to predict human estimates of roughness in texture photographs using measures 


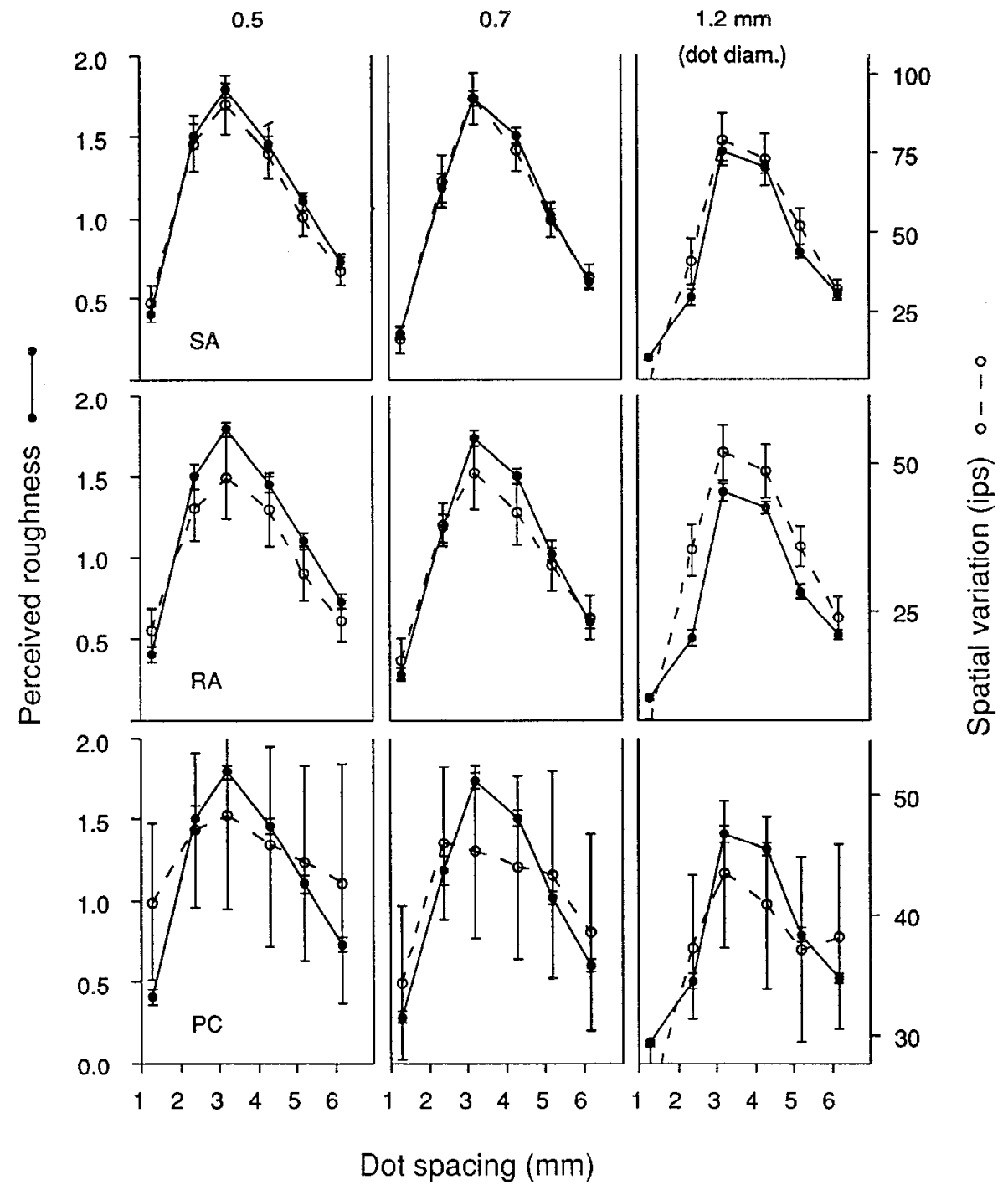

Figure 14. Two-point spatial variation and roughness versus dot spacing. Details are as in Figure 7.

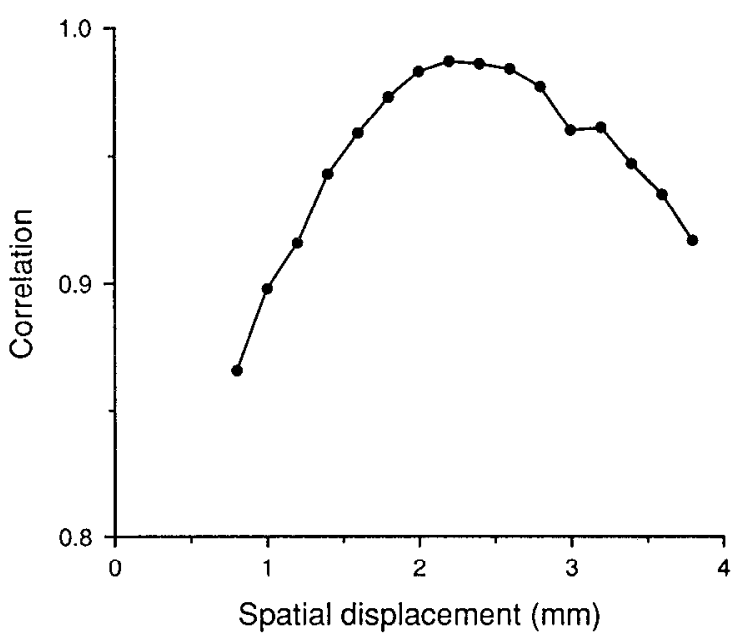

Figure 15. Correlation between roughness and SA 2-point spatial variation versus spatial displacement. Each point represents the correlation coefficient between subjective roughness and SA 2-point spatial variation based on the displacement between points specified on the abscissa. of brightness variability was unsuccessful (Tamura et al., 1978). As the authors noted, visual estimates of roughness may be based on highly derived notions of what a surface would feel like if it could be explored tactually.

In audition, roughness is an important and much-studied dimension. Of particular interest is the finding by Guirao and Garavilla (1976) that the perceived roughness of pure tones and narrow-band noise is sensitive to both the degree and the period of amplitude modulation. This is qualitatively similar to our findings in the somatosensory system. It is interesting to speculate that similar neural mechanisms might account for roughness perception in more than 1 modality.

\section{References}

Culbert SS, Stcllwagen WT (1963) Tactual discrimination of textures. Percept Mot Skills 16:545-552.

Cussler EL, Zlotnick SJ, Shaw MC (1977) Texture perceived with the fingers. Percept Psychophys 21:504-512.

Darian-Smith I, Kenins P (1980) Innervation density of mechanoreceptive fibers supplying glabrous skin of the monkey's index finger. J Physiol (Lond) 309:147-155.

Ekman G, Hosman J, Lindstrom B (1965) Roughness, smoothness 
and preference: a study of quantitative relation in individual subjects. J Exp Psychol 70:18-26.

Green BG (1981) Tactile roughness and the "paper effect." Bull Psychon Soc 18:155-158.

Guirao M, Garavilla JM (1976) Perceived roughness of amplitudemodulated tones and noise. J Acoust Soc Am 60:1335-1338.

Haralick RM, Shanmugan K, Dinstein I (1973) Textural features for image classification. IEEE Trans Syst Man Cybern 3:610-621.

Inukai Y, Saito S, Mishima I (1980) A vector model analysis of individual differences in sensory measurement of surface roughness. Hum Factors 22:25-36.

Johansson RS, Vallbo A (1979) Tactile sensitivity in the human hand: relative and absolute densities of four types of mechanoreceptive units in glabrous skin. J Physiol (Lond) 286:283-300.

Johnson KO (1983) Neural mechanisms of tactual form and texture discrimination. Fed Proc 42:2542-2547.

Johnson KO, Lamb GD (1981) Neural mechanisms of spatial tactile discrimination: neural patterns evoked by Braille-like dot patterns in the monkey. J Physiol (Lond) 310:117-144.

Johnson KO, Phillips JR (1988) A rotating drum stimulator for scanning embossed patterns and textures across the skin. J Neurosci Meth 22:221-231.

Johnson KO, Phillips JR, Hsiao SS, Fasman KF, Connor CE (1986) Neural correlates of tactile roughness perception. J Acoust Soc Am 79:S72.

Krueger LE (1970) David Katz's der aufbau der tastwelt (the world of touch): a synopsis. Percept Psychophys 7:337-341.

LaMotte RH (1977) Psychophysical and neurophysiological studies of tactile sensibility. In: Clothing comfort: interaction of thermal, ventilation construction and assessment factors (Hollies N, Goldman R, eds), pp 83-105. Ann Arbor: Ann Arbor Science.

Ledcrman SJ (1974) Tactile roughness of grooved surfaces: the touching process and effects of macro- and microsurface structure. Percept Psychophys 16:385-395.

Lederman SJ (1981) The perception of surface roughness by active and passive touch. Bull Psychonom Soc 18:253-255.

Lederman SJ (1982) The perception of texture by touch. In: Tactual perception: a sourcebook (Schiff W, Foulke E, eds), pp 130-167. Cambridge: Cambridge UP.
Lederman SJ, Taylor MM (1972) Fingertip force, surface geometry, and the perception of roughness by active force. Percept Psychophys $12: 401-408$.

Meenes M, Zigler MJ (1923) $\Lambda$ n experimental study of the perceptions of roughness and smoothness. Am J Psychol 34:542-549.

Mountcastle VB, LaMotte RH, Carli G (1972) Detection thresholds for stimuli in humans and monkeys: comparison with threshold events in mechanoreceptive afferent nerve fibers innervating the monkey hand. J Neurophysiol 35:122-136.

Phillips JR, Johnson KO (1981) Tactile spatial resolution: II. Neural representation of bars, edges, and gratings in monkey afferents. J Neurophysiol 46:1192-1203.

Phillips JR, Johnson KO, Hsiao SS (1988) Spatial pattern representation and transformation in monkey somatosensory cortex. Proc Natl Acad Sci USA 85:1317-1321.

Phillips JR, Johansson RS, Johnson KO (1990) Representation of Braille characters in human nerve fibers. Exp Brain Res 81:589-592.

Roscnfeld A, Kak AC (1982) Digital picturc processing, Vol 2. New York: Academic.

Sathian K, Goodwin AW, John KT, Darian-Smith I (1989) Perceived roughness of a grating: correlation with responses of mechanoreceptive afferents innervating the monkey's fingerpad. J Neurosci 9:12731279.

Stevens SS, Harris J (1962) Scaling of roughness and smoothness. J Exp Psychol 64:489-494.

Stone LA (1967) Subjective roughness and smoothness for individual judges. Psychonom Sci 9:347-348.

Talbot WH, Darian-Smith I, Kornhuber HH, Mountcastle VB (1968) The sense of flutter-vibration: comparison of the human capacity with response patterns of mechanoreceptive afferents from the monkey hand. J Neurophysiol 31:301-334.

Tamura H, Mori S, Yamawaki T (1978) Textural features corresponding to visual perception. IEEE Trans Syst Man Cybern 8:460-473.

Taylor MM, Lederman SJ (1975) Tactual perception of grooved surfaces. A model and the effect of friction. Percept Psychophys 17: 23-36. 